\title{
From the Bench to the Field in Low-Cost Diagnostics: Two Case Studies
}

\section{Citation}

Kumar, Ashok A., Jonathan W. Hennek, Barbara S. Smith, Shailendra Kumar, Patrick Beattie, Sidhartha Jain, Jason P. Rolland, Thomas P. Stossel, Catherine Chunda-Liyoka, and George M. Whitesides. 2015. "From the Bench to the Field in Low-Cost Diagnostics: Two Case Studies." Angew. Chem. Int. Ed. 54 (20) (April 27): 5836-5853. doi:10.1002/anie.201411741.

\section{Published Version}

doi:10.1002/anie.201411741

\section{Permanent link}

http://nrs.harvard.edu/urn-3:HUL.InstRepos:25045709

\section{Terms of Use}

This article was downloaded from Harvard University's DASH repository, and is made available under the terms and conditions applicable to Open Access Policy Articles, as set forth at http:// nrs.harvard.edu/urn-3:HUL.InstRepos:dash.current.terms-of-use\#OAP

\section{Share Your Story}

The Harvard community has made this article openly available.

Please share how this access benefits you. Submit a story.

\section{Accessibility}




\title{
From the Bench to the Field in Low-Cost Diagnostics
}

\author{
Ashok A. Kumar ${ }^{1}$, Jonathan W. Hennek ${ }^{1}$, Barbara S. Smith ${ }^{1}$, Shailendra Kumar ${ }^{2}$, Patrick \\ Beattie $^{2}$, Sidhartha Jain ${ }^{2}$, Jason P. Rolland ${ }^{2}$, Thomas P. Stossel ${ }^{3}$, Catherine Chunda-Liyoka ${ }^{4}$ \\ and George M. Whitesides ${ }^{1,5,}$
}

${ }^{1}$ Department of Chemistry and Chemical Biology, Harvard University, 12 Oxford St., Cambridge, MA 02138

${ }^{2}$ Diagnostics for All, 840 Memorial Drive, Cambridge, MA 02139

${ }^{3}$ Hematology Division and Center for Biomedical Innovation, Brigham and Women's Hospital, One Blackfan Circle, Boston, MA, 02115 United States

${ }^{4}$ Department of Paediatrics, University Teaching Hospital, Nationalist Rd., Lusaka, Zambia

${ }^{5}$ Wyss Institute for Biologically Inspired Engineering, Harvard University, 60 Oxford St., Cambridge, MA 02138

* Corresponding author’s email: gwhitesides@gmwgroup.harvard.edu 


\begin{abstract}
Despite the growth of research in universities on point-of-care (POC) diagnostics for global health, most devices never leave the laboratory. The processes that move diagnostic technology from the laboratory to the field - the processes intended to evaluate operation and performance under realistic conditions - are more complicated than they might seem. Two case studies illustrate this process: 1) the development of a paper-based device to measure liver function, and 2) the development of a device to identify sickle cell disease based on aqueous multiphase systems (AMPS) and differences in densities of normal and sickled cells. Technical and procedural lessons drawn from these experiences may be useful to those designing diagnostic tests for developing countries, and more generally, technologies for use in resourcelimited environments.
\end{abstract}




\section{Introduction}

One focus of lab-on-a-chip technologies has been the creation of point-of-care (POC) diagnostics. ${ }^{[1-3]}$ Publications describing these efforts have emerged at an exponential rate (Figure 1). Despite this activity, the promise of devices that will allow personalized and low-cost healthcare, to a large extent, remains just that: a promise. ${ }^{[4,5]}$ What steps are necessary to bridge the gap between publications on POC tests and fully developed POC tests that are actually used to improve healthcare?

Transitioning from a concept in a laboratory, to a product in the hands of users, is a difficult and expensive task in most fields, but it can become Herculean in medicine. Large clinical trials, and complex processes for regulatory approval, both require long times and substantial resources (both financial and human). As the first of several steps, a POC test must go from the bench to the field.

There is an argument, sometimes heard in universities, that academic scientists should be concerned only with discovering new methods and enabling new technologies, and that actual reduction to practice - including testing in the field — should be left to companies. This procedural model works (although imperfectly) when potential profits are large and risks are low. In these circumstances, market forces will sometimes encourage companies (either startups or large companies) to invest in the full development and commercialization of early-stage technologies. Technologies aimed for the bottom of the pyramid, ${ }^{[6]}$ however, do not fit this economic model because they often provide limited financial incentive and a high risk of failure in their intended application (often for reasons having little to do with the technology).

By performing their own initial field evaluations, academic scientists and engineers can improve their technologies, lower the costs of their further development, and encourage the 
Figure 1. The number of publications with the topic of point-of-care tests or diagnostics (Publications on Devices) and publications on the field evaluation of such devices (Field Trials) have both increased exponentially over the past decade. Publications on the devices themselves are about 60 times more frequent than publications about the field evaluation of the devices. Number of publications are based on results from the Web of Science (Thomson-Reuters) for the topic, (("point-of-care" AND (diagnostic OR test))), for "Publications on Devices," and for the topic, (("point-of-care" AND (diagnostic OR test)) AND ("field trial" OR "field evaluation" OR "clinical trial" OR "clinical evaluation")), for "Field Trials."

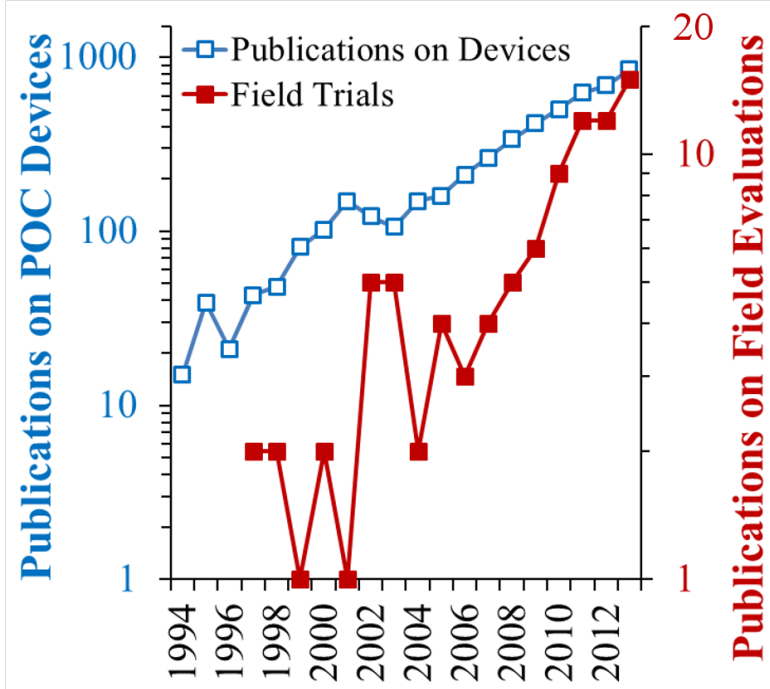

Year 
further investment needed to make a technology "real." This translational step is itself no small task, because it requires a set of skills and experiences that—while common in fields like public health — are rare in the academic departments of chemistry, biology, and bioengineering in which much of the advanced research in lab-on-a-chip technologies (and especially POC diagnostics) has been carried out.

Publications on field trials and evaluations of POC devices illustrate the difficulty of going from the bench to the field. Each year, only one paper is published about field testing or evaluation of POC devices for every 60 papers published about laboratory tests of devices aiming toward POC diagnostics. This ratio has been fairly consistent for the past two decades (Figure 1). Our objective in sharing our experiences in two representative technologies is to decrease this ratio by describing the less scientific, but remarkably interesting and difficult (both technically and procedurally) challenges that must be overcome to convert publications into a field-tested prototype.

Here we describe a general framework for creating POC diagnostics, and illustrate its structure with two case studies from our own experience. In the last seven years, we have taken two technologies from the bench ${ }^{[7-10]}$ to the field. ${ }^{[11,12]}$ Each technology, has required a different approach. In one case, development and field testing were done through a strong partnership with a non-profit company. In the other case, development and field testing were led by our academic laboratory. By sharing these details, we hope that others interested in proving and improving their devices can benefit from our experiences, and from the lessons we have learned, and can anticipate some of the challenges of the phase of development centered on field-testing a new technology that is ultimately intended for reality.

\subsection{What is a Field Trial?}


A scientist who has designed a new method to detect a disease in a simple, portable device might believe that the most appropriate way to test the device in the field would be to travel to rural clinics in low- and middle-income countries (LMICs) and to begin to use the devices with patients. If the device is designed for the POC, should not the POC be the best place to test the device? Testing the performance of a device at the POC is essential, but not necessarily the first work that should be done in the field.

"Field trials" and, more generally, "field work", refers to a wide range of activities

(Figure 2). Work in field settings can be broken down into four tiers: Tier 1) needs-identifying a problem and understanding its context; Tier 2) evaluation — - testing a prototype of a device in the field to identify aspects of the test to improve; Tier 3) validation - demonstrating clinical performance in a field setting; and Tier 4) efficacy — testing whether the use of the device has an impact on outcomes in health. The two cases we describe were at a stage between Tier 2 and Tier 3 when they were evaluated in the field. In both cases, however, field work began much earlier, and included an assessment of needs, and an evaluation of designs with potential endusers.

\section{A Framework for Development}

\subsection{Defining a Problem}

A successful technology must solve a real problem. For a diagnostic device intended for LMICs to make an impact, the problem addressed by the device should, generally, have four characteristics: 1) A substantial number of people should be impacted by the disease for a research program intended to ameliorate the problem to receive adequate attention from funding agencies, non-governmental organizations (NGOs), and governments. That is, it should ultimately be sufficiently important for some agency concerned with its amelioration to be 
Figure 2. The spectrum of field work can be broken down into four tiers. Progressing upwards from an initial assessment of needs, the activities and milestones that a device must pass requires increased time and resources. Field trials of a POC diagnostic for LMICs can refer to testing in an LMIC done anywhere above Tier 2. Field trials of the paper-based liver function test (LFT) and the density-based test for sickle cell disease (SCD-AMPS) were between Tier 2 and Tier 3.

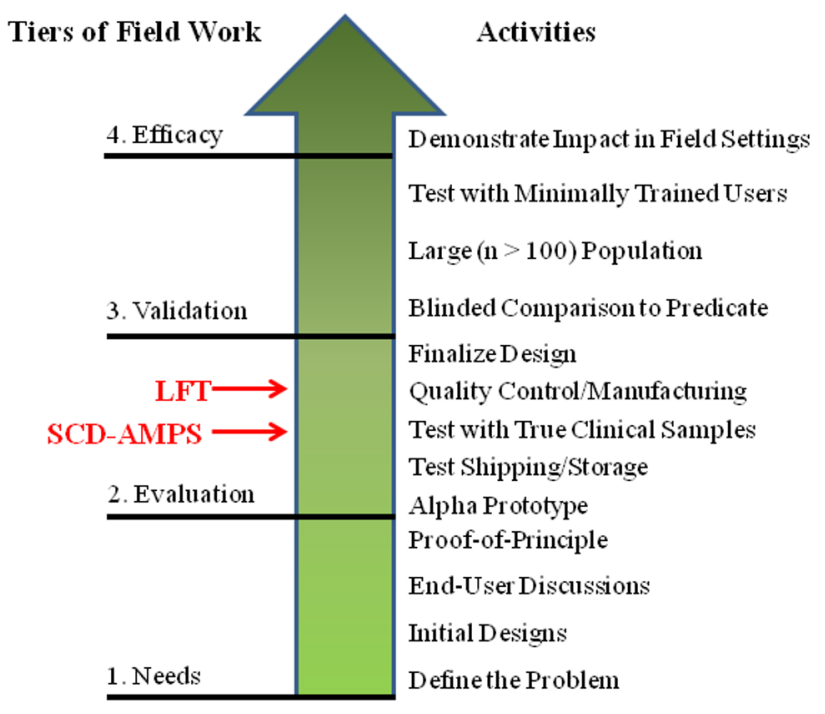


willing to implement (and help pay for) a successful solution. ${ }^{[13]}$ 2) The diagnostic device being designed should provide actionable information that can improve the well-being of the patient. 3) Simple interventions should already exist to treat the patient, or-for untreatable diseases (e.g., now, for example, dengue or Ebola) — to point toward a public health intervention once a diagnosis has been provided. 4) Existing solutions to the problem should be inadequate. The last criterion is especially important when the final goal is to create a useful product. Analyzing the market for current solutions provides an idea of the level of performance that must be surpassed to make a substantial impact. Several reviews provide detailed examples for these characteristics applied to different diseases. ${ }^{[1,3,14,15]}$

To identify needs and outstanding problems in global health, reports from the World Health Organization (WHO) and the latest list of Grand Challenges from the Bill and Melinda Gates Foundation (BMGF), provide only a starting point. Both organizations do extensive research on the ground to develop these lists, but by their nature, these lists neglect the details. In POC applications, the detail is where the devil lives.

To understand better the problems in resource-limited environments that could be addressed by technology, experience at the point-of-care is necessary, whether it be in rural villages in Africa, urban slums in India, or a forward-deployed military unit in a combat zone. When this experience is not directly available in a university laboratory, it is invaluable to find partners who understand these needs, and can explain them in the context of technical challenges. Once one has a team that brings together technical capabilities, medical expertise, and an understanding of field work, it is—in principle — possible properly to identify a problem where new technology could have an impact.

\subsection{Building a Team}


Creating an interdisciplinary team with global scope requires little more than searching for potential partners, sharing ideas, and valuing what every member brings to the discussion. For scientists and engineers in academic departments, attending symposia or lectures aimed at medical audiences, or simply emailing doctors and researchers at local hospitals or nearby schools of public health are easy ways to find partners who can guide capabilities toward real problems.

International collaborations can often be initiated without boarding an airplane. In the U.S. and Europe, many hospitals and medical schools have at least some doctors who have worked in LMICs. Many of these doctors operate, or work with, non-governmental organizations (NGOs) that may be a resource for conducting trials. In developing countries, Ministries of Health and local NGOs can be valuable partners.

Trust and mutual understanding are important aspects to any partnership, but especially so in international collaborations. Online communities and forums, such as Global Health Delivery Online (www.ghdonline.org) and Engineering for Change (www.engineeringforchange.org), make it much easier to begin communicating with potential partners, but establishing a good working partnership is often best done in person. There are a number of international conferences and workshops on POC diagnostics each year. Universities also offer courses that take students and professors abroad to work with organizations in the field. Taking advantage of these opportunities can lead to partnerships and new research programs. Partnering with organizations that have experience doing field work is another option. Engineers Without Borders (EWB) provides a grassroots network with global connections. Larger institutions like the Program for Appropriate Technologies in Health (PATH) and the 
Foundation for Innovative New Diagnostics (FIND) are particularly well-suited to help evaluate diagnostics. Table 1 lists these and other resources to help establish partnerships.

\subsection{Design Considerations for a Diagnostic Device}

A well-defined problem requires that solutions fit specific constraints. The ASSURED criteria (affordable, sensitive, specific, user-friendly, robust, equipment-free, delivered), developed by the $\mathrm{WHO},{ }^{[16]}$ provide a rough guide to, and check-list for, design of POC devices, but should be understood in the specific context under which they were developed: criteria for rapid tests for sexually transmitted infections in a world before ubiquitous cell phones, and in which electricity was less available than it is (in many locations) now. These criteria are important, but, as others have pointed out, they are guidelines and not a substitute for the considerations and requirements that are specific to a particular disease. ${ }^{[1]}$ Some problems require quantitative measurements, while others only need a simple yes/no answer. In some cases, the ASSURED criteria may be too constraining. For example, the "equipment-free" in ASSURED may not apply for devices to be used in a district hospital that supports small-scale equipment. Similarly, a cell phone could count as a piece of equipment, but simple mobile phones are now common, even in many remote villages. (More advanced cell-phones that are now often proposed for use in resource-limited, POC applications, often are not available. $)^{[17]}$

Those developing POC technology can visit sites where they intend their devices to function, or they can work with clinical and international collaborators to learn the detailed

context of their diagnostic target. ${ }^{[18,19]}$ Designing a device with a specific context and problem in mind avoids unnecessary restrictions, while recognizing constraints that are crucial to success in a field evaluation. 
Table 1. List of potential partner organizations with global reach.

\begin{tabular}{|c|c|c|c|c|}
\hline Organizations & Type & Headquarters & $\begin{array}{l}\text { Countries of } \\
\text { Collaboration }\end{array}$ & $\begin{array}{l}\text { Experience } \\
\text { Developing } \\
\text { Diagnostics }\end{array}$ \\
\hline Bill and Melinda Gates Foundation & NGO & $\begin{array}{l}\text { Seattle, WA, } \\
\text { USA }\end{array}$ & $>100$ & Yes \\
\hline $\begin{array}{l}\text { Center for Emerging \& Neglected } \\
\text { Diseases (CEND) }\end{array}$ & $\begin{array}{l}\text { University } \\
\text { Center }\end{array}$ & $\begin{array}{l}\text { Berkeley, CA, } \\
\text { USA }\end{array}$ & Worldwide & Yes \\
\hline $\begin{array}{l}\text { Center for Integration of Medical } \\
\text { Innovation and Technology (CIMIT) } \\
\& \text { Center for Global Health (CGH) }\end{array}$ & Consortium & $\begin{array}{l}\text { Boston, MA, } \\
\text { USA }\end{array}$ & $>20$ & Yes \\
\hline $\begin{array}{l}\text { Clinton Health Access Initiative } \\
\text { (CHAI) }\end{array}$ & Foundation & $\begin{array}{l}\text { Boston, MA, } \\
\text { USA }\end{array}$ & $>25$ & Yes \\
\hline D-Lab (MIT) & $\begin{array}{l}\text { University } \\
\text { Center }\end{array}$ & $\begin{array}{l}\text { Cambridge, } \\
\text { MA, USA }\end{array}$ & $>20$ & Yes \\
\hline $\begin{array}{l}\text { Engineers for a Sustainable World } \\
\text { (ESW) }\end{array}$ & Network & $\begin{array}{l}\text { Pittsburgh, PA, } \\
\text { USA }\end{array}$ & $>9$ & \\
\hline Engineers Without Borders (EWB) & Network & $\begin{array}{l}\text { Denver, CO, } \\
\text { USA }\end{array}$ & $>47$ & \\
\hline $\begin{array}{l}\text { Foundation for Innovative New } \\
\text { Diagnostics (FIND) }\end{array}$ & NGO & $\begin{array}{l}\text { Geneva, } \\
\text { Switzerland }\end{array}$ & $>60$ & Yes \\
\hline $\begin{array}{l}\text { Global Scientific Solutions for } \\
\text { Health, Inc. (GSSHealth) }\end{array}$ & Consultants & MD, USA & $\begin{array}{l}>15 \text { (Africa \& } \\
\text { Asia) }\end{array}$ & Yes \\
\hline John Snowe Inc. & Consultants & $\begin{array}{l}\text { Boston, MA, } \\
\text { USA }\end{array}$ & $>75$ & Yes \\
\hline $\begin{array}{l}\text { National Center for the Advancement } \\
\text { of Translational Science (NCATS- } \\
\text { NIH) }\end{array}$ & $\begin{array}{l}\text { Government } \\
\text { Institute }\end{array}$ & $\begin{array}{l}\text { Bethesda, MD, } \\
\text { USA }\end{array}$ & Worldwide & Yes \\
\hline $\begin{array}{l}\text { National School of Tropical } \\
\text { Medicine (Baylor) }\end{array}$ & $\begin{array}{l}\text { University } \\
\text { Center }\end{array}$ & Waco, TX, USA & $>7$ & \\
\hline Partners in Health $(\mathrm{PIH})$ & NGO & $\begin{array}{l}\text { Boston, MA, } \\
\text { USA }\end{array}$ & $>12$ & \\
\hline $\begin{array}{l}\text { Program for Appropriate } \\
\text { Technologies in Health (PATH) }\end{array}$ & NGO & $\begin{array}{l}\text { Seattle, WA, } \\
\text { USA }\end{array}$ & $>70$ & Yes \\
\hline Sandra Rotman Centre & $\begin{array}{l}\text { University } \\
\text { Center }\end{array}$ & $\begin{array}{l}\text { Toronto, ON, } \\
\text { Canada }\end{array}$ & Worldwide & Yes \\
\hline $\begin{array}{l}\text { Stanford Biodesign \& Center for } \\
\text { Innovation in Global Health } \\
\text { (Stanford) }\end{array}$ & $\begin{array}{l}\text { University } \\
\text { Center }\end{array}$ & $\begin{array}{l}\text { Stanford, CA, } \\
\text { USA }\end{array}$ & Worldwide & Yes \\
\hline $\begin{array}{l}\text { U.S. Agency for International } \\
\text { Development (USAID) }\end{array}$ & $\begin{array}{l}\text { Government } \\
\text { Agency }\end{array}$ & $\begin{array}{l}\text { Washington, } \\
\text { DC, USA }\end{array}$ & $>100$ & Yes \\
\hline
\end{tabular}




\subsection{Funding}

Finding funding for field trials can be a challenge: these activities fall in the wasteland between traditional academic research and corporate development. A first step is to understand costs. Depending on the size and scope of the field evaluation, costs can range from $\$ 15,000$ (e.g., a week-long program in a rural setting to get end-user feedback) to over $\$ 100,000$ (e.g., a six-month clinical evaluation of performance on several hundred subjects). By working with the international partners on the team and maintaining clear communications, one can estimate costs for personnel, equipment, and local transportation for a specific program. With a realistic set of costs, the team can factor field testing into budgets for grants from familiar funding agencies, like the NIH in the U.S. and the European Commission in Europe, or foundations sympathetic to global health causes, such as the BMGF or The Wellcome Trust.

Many institutions and non-profit organizations offer "accelerator," "innovation," or "translational" grants, designed to position a technology in such a fashion that it becomes easier to obtain funding from companies or venture capitalists. Design competitions or "hack-a-thons" are also becoming popular and can provide money, insight from others in the field, and a mechanism to build a team.

Funding may also be available from LMICs interested in technologies that will be of benefit to their citizens. Even when money is not available, in-kind services — such as access to space in a clinical laboratory or accommodations in dormitories - may reduce the amount of funds needed from external sources. Close connections to partners in the country can be crucial to take advantage of such opportunities. 
Companies that make diagnostic instruments and other supplies (e.g., Beckman Coulter and PerkinElmer) are sometimes willing to donate ancillary supplies and equipment when there is a potential benefit to the public; these kinds of donations can further reduce the costs of a field evaluation.

\subsection{When is a device ready for field work?}

Ideally, one would test a device in field settings early and often throughout the entire spectrum of invention, development and field testing (Figure 2). ${ }^{[20]}$ The constraints of time and funding, however, require a more judicious use of resources. Much preliminary work can be done in a laboratory or with medical partners in developed countries. The state of development that a device must reach before testing in the field depends on the specific problem being addressed, and the objectives of the field work. Diagnostic devices developed in academic laboratories can often benefit from an early, preliminary evaluation of operability in the field. Sometimes a device may benefit from a more in-depth field evaluation to identify operational and performance issues before advancing to validation (Tier 3). How can one tell whether a device is in a phase of development that will benefit from evaluation in the field?

A field evaluation of a prototype device (between Tier 2 and 3) can quickly identify the most critical weaknesses of the device. The effect of environmental conditions (e.g., temperature and humidity), variations in biological specimens (i.e., testing on true samples versus surrogates), and problems with use and interpretation are all critical challenges that can be identified. These kind of studies can be short ( $<1$ month) and require a more modest number ( $\mathrm{n}$ $\sim 30$ ) of subjects than a higher-tier field trial because the objective is to identify critical issues with the device rather than subtle influences. 
Despite the expectation of some area of failure, a high degree of confidence in the device is necessary to undertake a field evaluation. A proof-of-principle—demonstrations that the device works in a laboratory setting using clinically relevant samples or surrogates (e.g., serum or artificial urine spiked with an antigen) - provides a degree of confidence that, given proper settings and conditions, the device should work. Although initial validation on 30 or more samples is desirable to provide statistical power ${ }^{[21]}$ smaller sample sizes may still provide confidence in a result depending on the size of the effect being detected and the sensitivity required. As a minimum, however, one should not use less than seven independent samples. ${ }^{[21]}$

It is important to make sure that experiments with surrogate samples are independent, not just replications; ${ }^{[22,23]}$ for example, spiking different levels of an antigen into aliquots of plasma from the same sample would not provide the same amount of variation in the background as spiking antigen into plasma from different samples. If using surrogate samples, it is important to consult closely with a clinical specialist to understand limitations of the surrogate for the test that the device performs. Using a sample representative of the sample in the field provides the best test of a device. A device designed and tested only on blood from venipuncture may behave differently with fresh blood from a fingerprick. (Obtaining a reproducible and high quality sample from a fingerprick requires care and technique to avoid hemolysis or inclusion of large volumes of interstitial fluid). ${ }^{[24]}$

Testing the device with naive users - people who were not involved in the development of the device — can provide important information about design and operability of the device. Not only does a naive user offer feedback about the usability of the test, and a comparison with existing techniques/devices (if any), the performance of the test being run and interpreted by such a user provides a more realistic estimate of performance in the field than use and 
interpretation by the developers. With both of the technologies that we describe in this article, most testing before field work was done with some involvement of the developers of each technology. If naive users had been introduced in testing in the laboratory, perhaps we could have reduced the time spent on pilot trials, or identified areas to improve on the devices before they were evaluated in the field.

Devices designed to give a binary readout (i.e. "positive" or "negative") must be sensitive (able to detect positives) as well as specific (able to avoid classification of a negative sample as positive). If a threshold is used to define whether a measurement is classified as positive or negative, a receiver operating characteristic (ROC) curve can provide a simple visual tool to understand the performance of the test. ${ }^{[25]}$ If the device provides a quantitative measurement, comparison to measurements from a standard diagnostic test using a Bland-Altman plot can identify potential bias in the measurement. ${ }^{[26]}$

With confidence that the biological and technical side of the device can work, development efforts should focus on reducing the sources of confounding factors. In preparation for field evaluations, one needs to develop quality controls, identify suitable packaging, set storage requirements, and set shipping methods. Packaging devices, and then storing them in an oven or a high humidity environment, can provide quick tests for stability under extreme storage conditions. Packing some devices and sending them by a courier (e.g. FedEx, DHL, or UPS) with a return service provides exposure to different shipping environments. The degree to which all these factors are understood and accounted for sets the level of confidence that a device will perform as expected in a clinical trial, and provide a useful measure of performance.

Field evaluations conducted during this phase of development, before the final design has been frozen, provide an important opportunity to test a device on clinically relevant samples, and 
to identify unexpected problems, before conducting a field trial on the level of the third tier of field work (Figure 2), such as a trial for regulatory approval. In both of the case studies that follow, the design was not finalized, but work to test for shipping, stability, and quality control had been done to different degrees. Table 2 provides a representative example of a timeline for development and field evaluation.

A field trial at or above Tier 3 requires high confidence that the device will work, and a fixed design (including packaging and storage conditions). This level of confidence requires demonstration of diagnostic accuracy (both sensitivity and specificity) on larger numbers of clinically relevant samples $(n>30)$ on devices produced in different lots. Multiple users should both perform and interpret tests. The intention of a field trial at Tier 3 is no longer to troubleshoot the device, but to demonstrate validity of the rapid test (often as a means towards regulatory approval). A field trial above Tier 4 requires a device that has passed a field trial at Tier 3 and is essentially in the form of a product. At Tier 4, field work aims to establish whether the use of the device at the POC provides a significant health outcome. Above Tier 3, the support of a company is often critical to bring manufacturing standards and scale to validation and efficacy testing.

\subsection{Ethical Considerations}

Research involving human subjects generally requires the approval of an Institutional Review Board (IRB). IRBs are committees common at universities and hospitals; they review all research proposals involving human subjects to ensure that the study is designed ethically and participants are properly informed and protected. (An important exception relevant to diagnostics is the use of existing samples that are either publicly available or obtained in such a manner that subjects are unidentifiable.) In any case, researchers involved in a field evaluation 
Table 2. Representative timeline for moving a device from the laboratory to a field evaluation.

Timeline - 5 Year Plan

1. Defining the Problem

a. Team building

b. Needs assessment

2. Device Design \& Testing

a. Prototyping

b. End-user feedback

c. Validation on clinical samples

d. Quality Control/Stability/Storage

3. Supporting Activities

a. Grant Writing

b. IRB approvals

c. Institutional agreements

3. Field Evaluation

a. Trial Design

b. Shipment/Purchasing of Supplies

c. Training

d. Pilot Phase

e. Full Study

4. Next steps

a. Analysis of Results

b. Publication

c. Discussion with companies/providers

\begin{tabular}{|c|c|c|c|c|}
\hline 1 & 2 & 3 & 4 & 5 \\
\hline & & & & \\
\hline & & & & \\
\hline & & & & \\
\hline & & & & \\
\hline & & & & \\
\hline & & & & \\
\hline & & & & \\
\hline & & & & \\
\hline & & & & \\
\hline & & & & \\
\hline & & & & \\
\hline & & & & \\
\hline & & & & \\
\hline & & & & \\
\hline & & & & \\
\hline & & & & \\
\hline & & & & \\
\hline & & & & \\
\hline & & & & \\
\hline & & & & \\
\hline & & & & \\
\hline
\end{tabular}


should complete training on human-subject research. The IRB review process, while sometimes cumbersome, is essential to protect the subjects in a trial from physical or emotional harm. In fact, if approached properly, committees on the use of human subjects and IRB committees can provide invaluable guidance to ensure the ethical and proper collection of data. Often, these committees are knowledgeable about regulatory requirements, and they can provide advice to ensure that the study is designed in a manner appropriate for regulatory approval. In general, field trials carried out abroad must be approved both by an IRB in the country of the trial, and by a separate IRB in the country where the research project originates. Each IRB committee may have different requirements and standards; the process of reconciling these differences can take months.

\subsection{Designing a Study}

Once the prototype is ready to be tested in the field at or above Tier 2 (Evaluation), the design of the study becomes critical. With the medical and local team, one must lay out clear goals for the study. The entire team should know which tier of field work is expected because, as discussed earlier, the objectives and requirements for each tier differ significantly. During the design process, an institutional agreement between the research institution and the site of the field work should be established. This agreement can take the form of a subcontract or a memorandum of understanding. Clear, explicit expectations of work and commitments should be laid out, including precise language about financial commitments and oversight.

During a field evaluation, one may want to take a device to rural clinics to obtain feedback about the operation, interpretation, and design of the device. Such field work falls in Tier 1, and has different requirements for partners in LMICs and for the design of the study. A field evaluation, and an evaluation of usability at the POC, can be done concurrently, but each 
requires a specific set of goals and objectives. In some cases, they may each require separate approvals from the IRB.

Field evaluations past Tier 2, and before final efficacy testing (Tier 4), usually include checking the performance of the device on clinically relevant samples. Estimating performance requires comparison of measurements from the device to a "gold-standard test" (i.e., a widely accepted, standard clinical test). Often, these tests are not available at the POC. The requirement to compare results to a gold standard may mean that initial trials must be done in a regional hospital in-country, where the necessary equipment is available to perform a gold standard test, rather than at rural clinics. If one aims to characterize device performance, it is essential to follow best practices ${ }^{[27,28]}$ and sound statistics ${ }^{[29]}$ (e.g., ensure adequate sample sizes for statistical power, ${ }^{[30]}$ proper blinding of samples, ${ }^{[27]}$ and well-defined inclusion and exclusion criteria $^{[27]}$ ).

Recruiting and training staff to carry out the study can require weeks to months; the investment in time used to find qualified and committed staff members will allow a study to be more robust against unexpected external events. Training requires clear instructions on recruitment, workflow, sample collection and distribution, carrying out the rapid test, carrying out the standard test, and recording results. PATH and other organizations can sometimes help expedite this process at established sites. In addition to time for training, time should be set aside for a pilot study before the main study begins. A pilot phase allows logistical problems to be identified and remedied without compromising the quality of the data from the trial.

Instruments used for the collection of data (i.e., questionnaires and laboratory logs) should include as much information as possible without becoming cumbersome. Moving from the lab to the field introduces many variables; unless these externalities have been tracked, the 
results of the study may be difficult to interpret. Events that could affect a device occur from the moment devices are prepared in the laboratory to the time that they are used, but some factors are not immediately apparent. For example, including a temperature logger when devices are shipped is often overlooked (indeed, see the Challenges section of the second case study).

\subsection{Context and Culture}

Establishing a working relationship with partners overseas early in the development of a device reduces the risk of a misunderstanding later, during the implementation of a field trial or evaluation. Partners may not have protected research time, especially in clinical settings, and their work load may prevent them from devoting as much time to run the study as needed. In such cases, it may be appropriate to hire a dedicated study coordinator for the project. Different countries have different hierarchical structures. Understanding the local culture can prevent a social faux-pas that can undermine a study or put a partnership at risk.

\subsection{Challenges}

With all the complications of carrying out an international collaboration and field evaluation, unexpected challenges will inevitably arise. Strikes, natural disasters, and regional instability are just a few examples of unrelated events that can threaten a project. The project must have timelines, but be flexible. Each obstacle taken in stride is an indication that the device is making its way toward something useful.

\section{Case Study 1: Liver Function Test}

\subsection{The Problem}

Efforts to combat HIV have enabled access to anti-retroviral therapy (ART) in LMICs. As of 2013, over 10 million people were being treated with ART. ${ }^{[31]}$ Drug-induced liver injury (DILI) is a significant side effect associated with ARTs. DILI associated with nevirapine-based 
ART — widely used in the developing world — is of particular concern; rates of nevirapineassociated hepatotoxicity (a type of liver damage) exceed 13\%. ${ }^{[32,33]}$ Monitoring liver function provides an important tool to manage ART; ${ }^{[33,34]}$ dosages and treatments can be adjusted if signs of liver damage or hepatitis appear. Tests to monitor liver function, however, are often unavailable in low-resource settings where many patients with HIV receive care. Levels of serum transaminases (aspartate aminotransferase, AST, and alanine aminotrasferase, ALT) provide a standard for monitoring DILI, but generally require centralized labs and venipuncture. $^{[35]}$

\subsection{The Test}

AST and ALT are concentrated in the liver in hepatocytes and usually exist in low concentrations (5-40 units/L for AST and 5-35 units/L for ALT) in serum. If injury, toxicity, or inflammation of the liver result in damage to hepatocytes, these cells will secrete larger concentrations of these transaminases into the serum. The ratio of AST to ALT can be helpful in assessing the cause of liver injury; alcohol-related liver injury, for example, often results in a greater than 2:1 ratio of AST:ALT ${ }^{[35]}$ By choosing enzymes as markers for liver injury-in this case transaminases - we could create a colorimetric assay by providing a substrate specific to each enzyme that would result in a change in color. We describe the chemistry of the detection for AST and ALT in detail elsewhere. ${ }^{[9,36]}$

\subsection{The Team}

We built a team combining academia, industry, and medicine. A business plan competition at the Harvard Business School brought together business students and scientists from the Whitesides group. The Whitesides group had developed the initial idea of 3D paper 
microfluidic devices that formed the technical starting point for the development of the test for liver function. ${ }^{[7,36-39]}$

Diagnostics for All Inc. (DFA) was formed as a non-profit (501-c-3) engineering organization to develop paper-based devices—or indeed, any type of device that suits a task —as diagnostics. Academic groups excel at basic research and innovation, but usually lack the proper resources, incentives, motivation, and experience to do the detail-oriented engineering required to execute trials, regulatory clearance, manufacturing, and quality-control for a product. A company provided a vehicle to do high-quality engineering. The decision to create a non-profit company —DFA—stemmed from the idea that a for-profit entity driven by investors interested in financial return might be forced to focus on developed world applications of paper-based diagnostics rather than first to apply the technology to address needs in the developing world. (Whether a for-profit or not-for-profit model ultimately is more effective for POC diagnostics intended for resource-limited environments remains to be seen. The development of a test for HIV by Sia (at Columbia) and Linder (at Claros, now OPKO) provides an alternative example. ${ }^{[40]}$ Chin, Linder, and Sia also provide a helpful review of many of the companies working to commercialize POC diagnostics and their different funding structures. ${ }^{[4]}$ )

The DFA team identified the need for a POC liver function test early on, and discussions with doctors in Boston-area hospitals and experts in public health confirmed the importance of the problem, especially in countries with large populations of patients being treated for HIV or TB. After initially considering seven different assays, the team settled on ALT and AST.

Bioengineers from DFA developed a robust assay and integrated sample acquisition, preparation, and evaluation into a single device. Dr. Nira Pollock at Beth Israel Deaconess Medical Center (BIDMC) provided medical guidance and led clinical validation in Boston. Dr. 
Pollock identified a site in Vietnam to evaluate the test in a field setting. After initial validation, DFA called Bernhard Weigl, the director of NIH-funded Center for Point-of-Care Testing at PATH, for assistance in running a field evaluation. (We mention by name some of the specific individuals who were particularly important in developing this test, to emphasize the number and variety of people, skills, and connections necessary to accomplish field testing.)

\subsection{Designing a Solution}

To monitor DILI at the POC in LMICs, we created a low-cost, rapid, liver function test (LFT) for serum transaminases using paper-based microfluidics. ${ }^{[9,36]}$ Paper provides an inexpensive substrate for immunoassays. Patterning paper allows multiplexed flow, and small test zones; the latter reduce the need for large volumes of expensive reagents. Paper-based microfluidic devices provide an attractive system to develop POC tests for use where low cost is a key design criterion. Tests that are performed frequently, and potentially at high volume (e.g., tests to monitor liver function), are particularly sensitive to cost.

The specific problem of measuring liver function provided guidance in the design of the test. Although standard tests for serum transaminases provide a quantitative measurement, these measurements are generally interpreted in three bins. In practice, this binning means that a rapid test needs to provide a semi-quantitative readout that will allow results to be placed into the three bins, but further precision is unnecessary. To meet this requirement, we made a colorimetric test with a read-guide (a standard color bar) that would allow users to provide a semi-quantitative measurement of each test (Figure 3). Benchmark values for ALT and AST are based on measurements in blood serum. In order to measure transaminases from serum, we incorporated a plasma-separation membrane (PSM) into the paper device. 
With end-users at the POC in mind, the device was designed to be simple to use as possible, and interpretable by eye. The device was covered in plastic lamination to protect the paper test zones from the outside environment, and a small hole in the lamination over the PSM provided an entry to add blood directly from a fingerprick. Positive and negative controls built into the device provide a metric indicating if test results are valid (e.g., reagents are working, blood has not lysed). Several iterations of design incorporated improvements to the ease-of-use, sensitivity, and interpretation of the test (Figure 4).

\subsection{Validation and Preparation for Field Evaluation}

We tested analytical, operational and clinical performance of the LFT device. ${ }^{[9,36]}$ Briefly, these studies included defining the limits of detection, assessing repeatability, checking for crossreactivity and interference, optimizing the time for the assay, designing methods for metering the sample, comparing the performance of the device to gold-standard methods, and testing the environmental stability of the device. These studies were intended to validate the device and provide adequate supporting data to warrant initiation of field testing.

During this time, members of the team from DFA had opportunities to travel internationally for conferences and meetings with potential end-users in different healthcare settings. A trip to India in 2010 allowed a DFA member to show the design of the LFT at the time (Figure 4: Iteration 2) to clinical laboratory workers. Workers at the clinic felt that the test was too small, and demonstrated that ceiling fans in the hot environment had the potential to cause samples to evaporate and change the dynamics of the test. Later iterations of the device increased the size and added a small white tab make the test easier to hold (Figure 4: Iteration 3 onward). Protocols for running the test were modified to include placing the tests under a glass dish to prevent excessive evaporation. 
Figure 3. The design of a paper-based LFT provides a semi-quantitative test for serum transaminases. (A) The stamp-sized devices receive a sample of blood on the "application side" and provide a colorimetric readout on the "read side." (B) The entire process of running the test requires minimal sample manipulation. (C) Valid results are interpreted and binned into three levels based on elevation over the upper limit of normal (ULN) using a read guide.

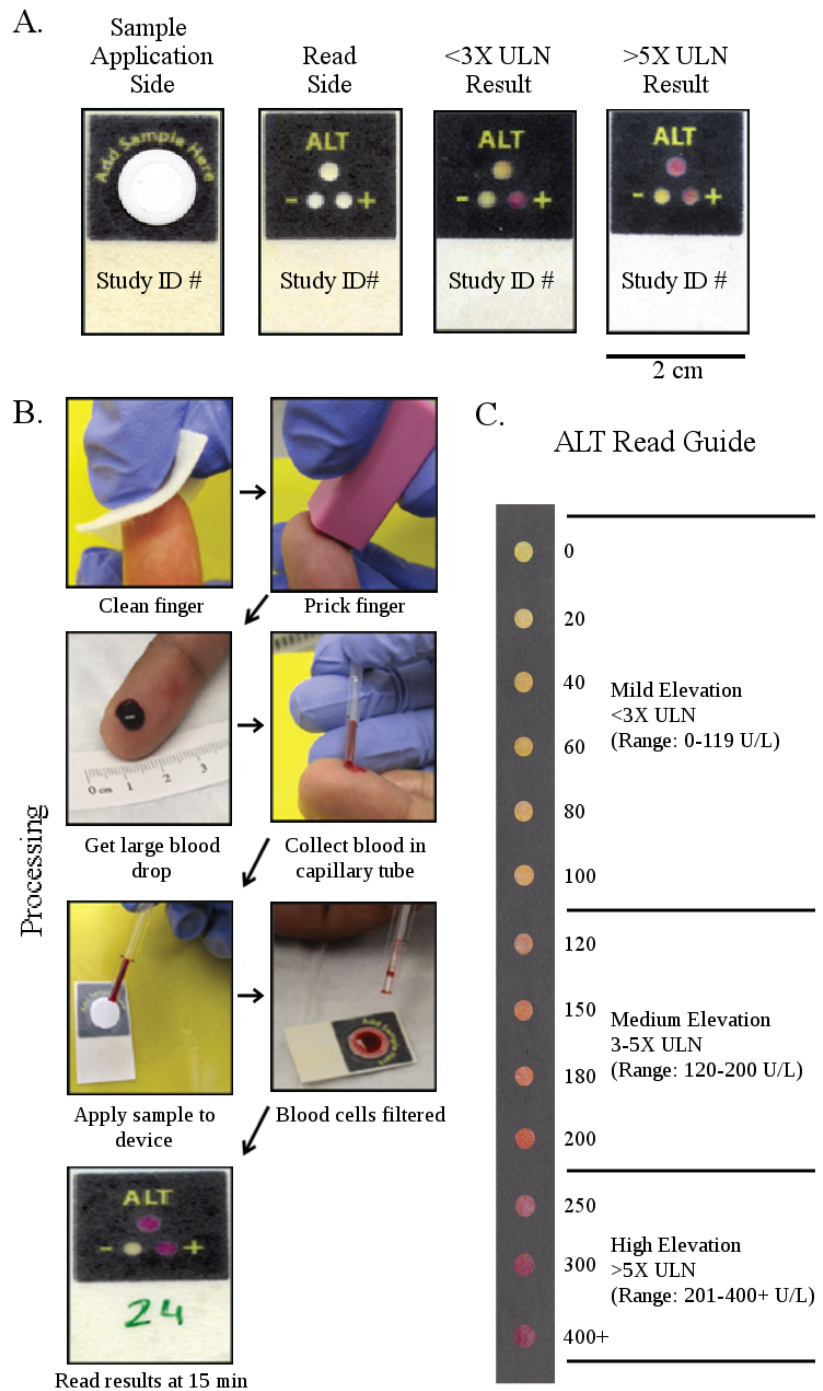


Figure 4. Iterations on the design of a rapid LFT (1-5). Each design has the same scale. The characteristics of each iteration demonstrate improvements to the design to create a user-friendly device.

Design Characteristics

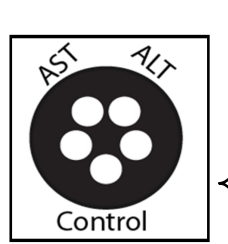

Strengths:

- Colorimetric assay

- Low-cost

- Incorporates plasma separation membrane

Weaknesses:

- No positive control for AST

- AST and ALT assays produce different colors and require separate read guides

2

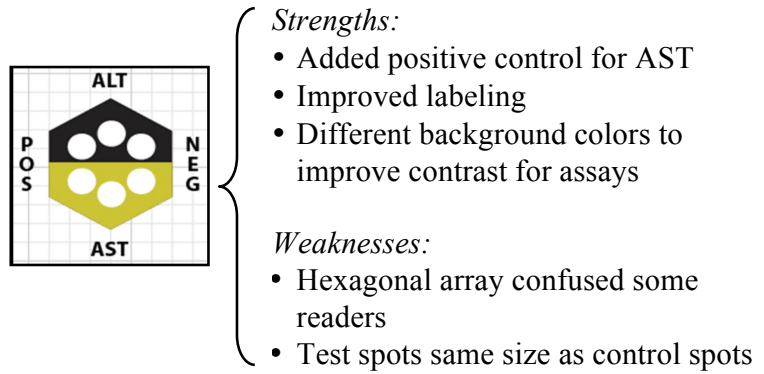

3

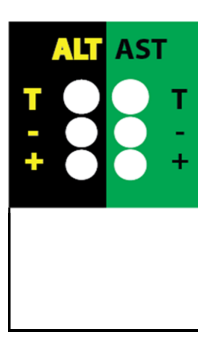

Strengths:

- Clear differentiation of AST and ALT

- Improved labeling

- Improved contrast with background

- Increased size of test zone (T) to emphasize importance over controls

Weaknesses:

- AST assay not reliable at high temperatures in pilot study
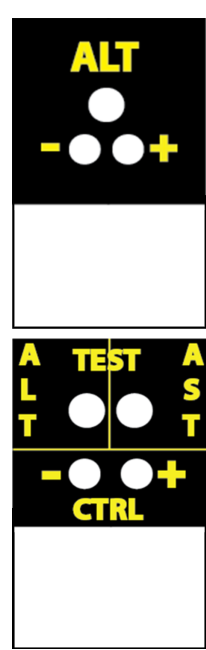

Strengths:

- Simplified readout

Weaknesses:

- No capability to measure AST

Strengths:

- Assay for AST and ALT now uses same chemistry

- Same color readout

- Allows same controls

- Multiplexing

- Simplified layout 
After three years of development, the team decided to perform a field evaluation to produce high-quality data for use in refining the device. Field evaluation would also assist in freezing aspects of the design that worked well; this step would then enable future trials for regulatory validation and enable manufacturing to be scaled up. Most importantly, we hoped the field study would provide a test to see if the device could perform as a POC diagnostic in a setting where it could have an impact on healthcare.

\subsection{Funding the Trial}

The initial work to develop the test on paper-based microfluidics was supported by a grant from BMGF. This grant also enabled some early travel to countries where a large number of patients on ART could benefit from a test to monitor DILI. Dr. Pollock was supported by an NIH K23 grant to carry out the clinical validation in Boston. By working with PATH, we were able to fund the field evaluation. PATH had an NIH funded Center to Advance Point-of-Care Diagnostics for Global Health.

\subsection{Study Design}

To obtain sufficient data, we wanted to enroll 600 patients in a controlled field setting. Working with BIDMC, we sought an international partner who worked with a large number of patients on ART and who would be able to benefit from a POC LFT. The partner also needed to have the infrastructure in place to measure serum transaminases as a gold standard comparison to our test. BIDMC had a long-standing partnership with hospitals in Vietnam through the Harvard AIDS Initiative in Vietnam (HAIVN). Dr. Pollock connected with Dr. Donn Colby who had spent years living and working with hospitals in Vietnam as part of this program. Dr. Colby provided an essential link between the medical team developing the device, and the local site in 
Vietnam where the device would be connected. With Dr. Colby's assistance, the Hospital for Tropical Diseases (HTD) in Ho Chi Minh City was identified as a site for testing.

The HIV clinic at the HTD provided the right combination of appropriate patient population and supporting infrastructure. The clinic we chose saw 3,000 HIV-positive patients per year who received ART. Of these, a significant proportion were on nevirapine-based ART (known to confer risk of DILI) or were at risk for co-infection with hepatitis B (HBV, 15\% prevalence) and/or hepatitis C ( $\mathrm{HCV}, 25 \%$ prevalence) — both of which can cause damage to the liver and could benefit from monitoring liver function. The clinic also had an existing practice of routine transaminase monitoring (once every six months) for patients receiving HIV treatment. As a result, the clinic had capabilities to do standard tests, but also could benefit from a POC LFT that could enable more frequent and less expensive monitoring.

The study recruited patients scheduled for routine clinical ALT testing (specimen collected by venipuncture) by their physicians; after venipuncture, subjects proceeded to fingerstick collection for LFT testing. Working with PATH and HTD, DFA and Dr. Pollock chose a target population of adults since this population would be one of the largest to benefit from the LFT and would be able to provide informed consent themselves (an important consideration to simplify IRB approval and the consenting process). The Vietnam field study was evaluated and approved by the Institutional Review Boards of HTD as well as by Research Ethics Committees at PATH and BIDMC. All participants in the study provided informed consent.

Working with HTD rather than a rural clinic enabled validation of results using standard tests. Using resources at HTD, automated ALT testing was performed in parallel (Roche Cobas 6000 analyzer) using blood obtained by venipuncture. In order to collect additional information 
that could be relevant to the performance of the devices, the study was designed to collect more clinical information, as available, for each subject: hepatitis B virus (HBV) status, hepatitis C virus (HCV) status, current HIV medications, current TB medications, and most recent CD4 count. Results of any laboratory tests ordered concurrently with ALT on the day of enrollment were also captured as available (e.g., AST, hemoglobin, hematocrit, platelet count, creatinine, and CD4).

Outside of clinical information, we also tried to record other variables that could influence the performance of the LFT. Packaged LFTs were stored in ambient conditions, and, thus, a temperature and humidity logger, combined with historical weather data from Weather Underground (wunderground.com) provided a log of ambient environmental conditions. Initially, no data loggers were included during the shipment of LFTs to Vietnam. The team carrying out the trial, however, added data loggers to the second batch of LFTs that were sent to the study site.

Three activities occurred at the site of the study: 1) training, 2) pilot phase, 3) study phase. The training was meant to familiarize all staff with the study and establish competency reading the LFT. The pilot phase was designed to recruit 50 subjects and ensure that study procedures were working as expected before beginning the study phase and recruiting 600 participants over six months.

\subsection{Implementation}

Approximately one month prior to the beginning of the study, representatives from PATH and DFA traveled to HTD and provided training to nurses assigned to carry out the study. The training curriculum included review of the study objectives and recruitment procedures, 
overview of the device structure and function, steps for completing the fingerstick and transferring sample to the device, and practice reading with mock devices. In addition, nurses were specifically instructed to read and record device results privately, without interaction with any other individual. The study nurses were required to pass a proficiency test using the mock devices (pass criteria: $>80 \%$ bin placement accuracy and $100 \%$ determination of invalid tests) before patient enrollment could start. If they failed this test, they were retrained with the mock devices and given another test. Each nurse was allowed a maximum of two attempts to pass the test. During the pilot phase, the study nurses received immediate feedback on correct and incorrect use (including fingerstick, sample transfer procedure, and device reading) from an expert DFA representative. No additional training or feedback was given once enrollment for the study began.

The temperatures during the pilot phase were often higher than the range for which the LFT was designed $\left(4-30{ }^{\circ} \mathrm{C}\right)$. The read-time for the test was adjusted for the higher temperature and the AST test was removed from the device due to poor stability of reagents for that test at higher temperatures (current improvements in the AST test have addressed these issues and will be evaluated in future trials).

All tests were performed following a set of instructions provided with each product by DFA, the details of which are described elsewhere. ${ }^{[1]}$ Notably, each test was read by two nurses. Neither the patients nor their doctors were informed of the results of their fingerstick testing. Although the field work took only six months, the total time to plan for the study, obtain IRB approval and carry out the work at the field site was greater than a year.

\subsection{Context and Culture}


Working with multiple partners - academics (Harvard and BIDMC), a non-profit company (DFA), an NGO (PATH), and a hospital (HTD)—provided the benefit of specialized knowledge and experience from each partner. Managing such a multi-institutional collaboration, however, also has challenges. Each partner has its own needs and its own timeframe. Academics want to publish results, hospitals need to maintain sufficient staff for their primary operations outside the study, companies want to learn as much as possible about their test for use in development, and NGOs needs to ensure that the device is at a sufficiently mature stage to merit investing time and resources into a trial. In the case of the field trial in Vietnam, we believe everyone met their needs effectively, but this success required clear, open, and frequent communication at the outset, and throughout, the study. For example, DFA and PATH had wanted to have ten nurses reading tests independently in order to estimate concordance for the visual test. HTD was not willing to provide that many nurses to the study based on the resources they had available. A compromise was reached in which HTD provided three nurses for the study. Rather than DFA or PATH hiring nurses, HTD received funds as a contractor, and they assigned nurses to the study.

Every healthcare system operates differently, and differences in norms have the potential to disrupt a study. At HTD, nurses rotate their positions every six months. The team from PATH and DFA arrived and began training nurses during the pilot phase in the middle of a rotation cycle; all the trained staff would have been rotated out of the clinic halfway through the study and could have caused major inconsistencies. The practice of rotating staff came to the attention of the PATH trainers, fortunately, during the pilot phase. Working with HTD, they were able to negotiate for some of the key trained personnel to remain at the clinic for the duration of the trial. 
Stigma associated with HIV can pose a challenge for diagnostics designed for those with the disease. Working with an established HIV clinic provided an environment where patients were comfortable. Sometimes patients may be reluctant to participate in a study when there is no direct compensation. Working with an HIV clinic where patients were already being monitored regularly for DILI, minimized the need to explain the potential future benefit of a rapid LFT.

\subsection{Challenges}

The study identified a number of parameters of the LFT paper chip that could be improved. Some involved ease-of-use (for example, the training required for accurate bin placement). Others related to quality control in components (a number of tests were invalid because a batch of plasma separation membranes was faulty). Even though DFA tested the influence of environmental conditions (humidity and temperature) on shelf-life, the results from the study indicated that further optimization of stability (of reagents, surprisingly, rather than enzymes) required improvement. Temperatures at the study site went up to $36{ }^{\circ} \mathrm{C}$ during the course of the study. These temperatures were higher than those recommended for storage.

At the beginning of the study, nurses felt that devices were difficult to read; by the end of the study, with familiarity, they reported that tests were easy to read $90 \%$ of the time, and that the instructions provided with the test were "very clear and easy to follow." The most frequently mentioned challenge to using the test was matching the color on the device to the color bar in the guide for the reader (Figure 3).

\subsection{Lessons learned}

Field evaluation of tests identified several aspects that required improvement. For example, improving bin placement required expanding the dynamic range of the assay to give 
clearer visual differences in color between different levels of ALT. Minimizing invalid tests required increased quality testing of the commercial materials used in the assembly of the LFT, and tightening the requirements for the controlled manufacturing environment to reduce lot-tolot variability. Stabilization of the reagents stored on the device was also required to improve shelf-life.

Like many rapid tests, the LFT requires that results be read within a specific window of time to avoid false positives and false negatives. In discussions with the nurses using the LFT in Vietnam, several expressed the understandable desire that tests should be less sensitive to time; ideally, they wanted tests that could be read over an interval of several hours after the assay had completed. As busy nurses in a hospital, it was difficult for them to manage time between patients and other duties, and to be available to read tests at very specific times.

Although POC diagnostics using paper-based microfluidic devices have been explored extensively in academic laboratories, this study was the first large-scale field evaluation of a device of this type. As such, it provided evidence that paper-based devices could be used practically in LMICs. In particular, it established that healthcare workers performing tests on samples from a clinically relevant population agreed well in their evaluation of visual readings. Successful development of this device for clinical use will, nonetheless, require further, iterative, optimization, but these initial field evaluations provided the guidance necessary for further development.

\subsection{Next Steps}

The newest prototypes have better accuracy, less sensitivity to environment conditions, and more importantly, a shelf life of greater than a year at $20-30{ }^{\circ} \mathrm{C}$. Most of the issues identified in Vietnam have been addressed. We are currently preparing for field testing of the new 
prototype and beginning the process of validation of the product for regulatory approval (CE mark).

The intensive training of users allowed in the context of this first field study is unlikely to be feasible or reproducible outside of study contexts. A thorough understanding of the minimal training requirements for novice users will ultimately be key to understanding the range of clinical environments in which this test can be used-whether that be in centralized clinics and performed by trained staff, decentralized clinical settings and performed by minimally trained health-care workers, or even at home and performed by patients themselves. DFA is currently planning an "Untrained User Study" with 50-100 participants in order to obtain Clinical Laboratory Improvement Amendment (CLIA) waived certification for the LFT device.

\section{Case Study 2: Sickle Cell Diagnostic Test}

\subsection{Problem}

Sickle cell disease (SCD) is an illness where an early diagnosis can have a major impact on health outcomes. Each year, over 300,000 children are born with SCD, most in sub-Saharan Africa and India. ${ }^{[41]}$ In countries without early diagnosis and treatment, the mortality rates of children under 5 years old with the disease are $50-90 \%{ }^{[42]}$ Interventions as simple as prophylactic penicillin and parental education can have a significant impact on child survival rates. ${ }^{[43]}$ The lack of a low-cost, rapid, point-of-care test for SCD, however, means that the potential of these interventions goes largely unrealized.

\subsection{The Test}

The distribution of densities of red blood cells in SCD is heterogeneous compared to red blood cells from a healthy individual. ${ }^{[44,45]}$ Red blood cells with a normal morphology have a maximum density of roughly $1.10 \mathrm{~g} / \mathrm{cm}^{3} \cdot{ }^{[45,46]} \mathrm{In} \mathrm{SCD}$, sickling and dehydration can lead to the 
formation of cells $\left(\rho>1.12 \mathrm{~g} / \mathrm{cm}^{3}\right)$ that are denser than the most dense red blood cells in a healthy individual. ${ }^{[44,45,47,48]}$ We developed aqueous multiphase systems (AMPS)—mixtures of polymers in water that spontaneously separate into immiscible liquid phases - to separate and visually identify the presence of dense cells characteristic of SCD.$^{[10,12]}$ The densities of the phases of AMPS provide a step-gradient in density. We designed a two-phase system ( $\rho_{\text {top }}=$ $\left.1.078 \mathrm{~g} / \mathrm{cm}^{3} ; \rho_{\text {bot }}=1.129 \mathrm{~g} / \mathrm{cm}^{3}\right)$ and a three-phase system $\left(\rho_{\text {top }}=1.077 \mathrm{~g} / \mathrm{cm}^{3} ; \rho_{\text {mid }}=1.108\right.$ $\left.\mathrm{g} / \mathrm{cm}^{3} ; \rho_{\mathrm{bot}}=1.120 \mathrm{~g} / \mathrm{cm}^{3}\right)$ such that only dense cells characteristic of SCD would be able to sink through the bottom phase (most dense) and form a visible red layer at the interface between the bottom phase and the seal at the bottom of the container. We summarize the details of the test elsewhere. ${ }^{[10,12]}$

\subsection{The Team}

The Whitesides group had developed aqueous multiphase systems (AMPS) - mixtures of polymers in water that spontaneously form immiscible liquid phase — as a method to separate cells by density. ${ }^{[10,49]}$ A chance encounter with Dr. Thomas Stossel (Brigham and Women's Hospital, Boston) provided the incentive to think seriously about SCD. Dr. Stossel had spent the better part of a decade doing medical and dental work in rural Zambia. As a hematologist, he knew the burden of undiagnosed patients with SCD in that country. He also understood the constraints that a test would have to meet to be useful in rural areas.

Once the team realized the potential impact of a density-based assay to identify sickle cell disease, they met with Dr. Carlo Brugnara (Children's Hospital, Boston). Dr. Brugnara added a depth of understanding of the density of red blood cells in SCD. ${ }^{[50,51]}$ Dr. Stossel connected the team to Dr. Catherine Chunda-Liyoka, a physician at the University Teaching Hospital (UTH) in 
Lusaka, Zambia. Dr. Chunda-Liyoka complemented Dr. Stossel's knowledge of the needs for sickle cell diagnostics with her experience managing SCD in patients in Zambia.

The team moved from a conceptual idea to an evaluation in the field in Zambia in three years (Table 3). The speed of development was driven in part by the simplicity of the technology, and by the ability of team members to work together efficiently in the design and implementation of the field work.

\subsection{Design considerations}

Using AMPS, we generated self-assembling, thermodynamically stable, step-gradients in density designed to separate dense cells present in SCD, and provide a visual test. ${ }^{[10]}$ The stepgradients that form in AMPS on settling in gravity, or - more rapidly - centrifugation, allowed us to make large batches of these mixtures of polymers and preload them into microhematocrit capillary tubes. The density of red blood cells can change in response to the characteristics of the medium (e.g., pH and osmolality). We, thus, designed our initial tests to match physiological $\mathrm{pH}(7.40 \pm 0.02)$ and osmolality $(295 \pm 15 \mathrm{mOsm} / \mathrm{kg}) .{ }^{[52]}$ (Other values of these parameters may also be useful for a rapid test, as long as they are controlled. Changing either value may require adjusting densities to reflect the shifts in density that can occur in the population of red blood cells.)

Discussions with Dr. Stossel and Dr. Chunda-Liyoka indicated that it was important to minimize the requirements for power and time for the test, so that it could be run in rural clinics. Centrifugation is necessary for cells to move through an AMPS in the short time (minutes rather than hours) required. The faster the centrifuge, the more the power that is required, but the shorter the time that is needed for the test. After consultation, we concluded that shortening time was more critical than reducing the use of electricity, and that a target of less than 20 minutes 
Table 3. Timeline from the conceptualization of a density-based rapid test for sickle cell disease to a field evaluation in Zambia.

\section{Timeline}

\section{Task}

\section{Defining the Problem}

a. Team building

b. Needs assessment

\section{Device Design \& Testing}

a. Prototyping

b. End-user feedback

c. Validation on clinical samples

d. Quality Control/Stability/Storage

\section{Supporting Activities}
a. Grant Writing
b. IRB approvals
c. Institutional agreements

\section{Field Evaluation}
a. Trial Design
b. Shipment/Purchasing of Supplies
c. Training
d. Pilot Phase
e. Full Study

\section{Next steps}

a. Analysis of Results

b. Publication

c. Discussion with companies/providers

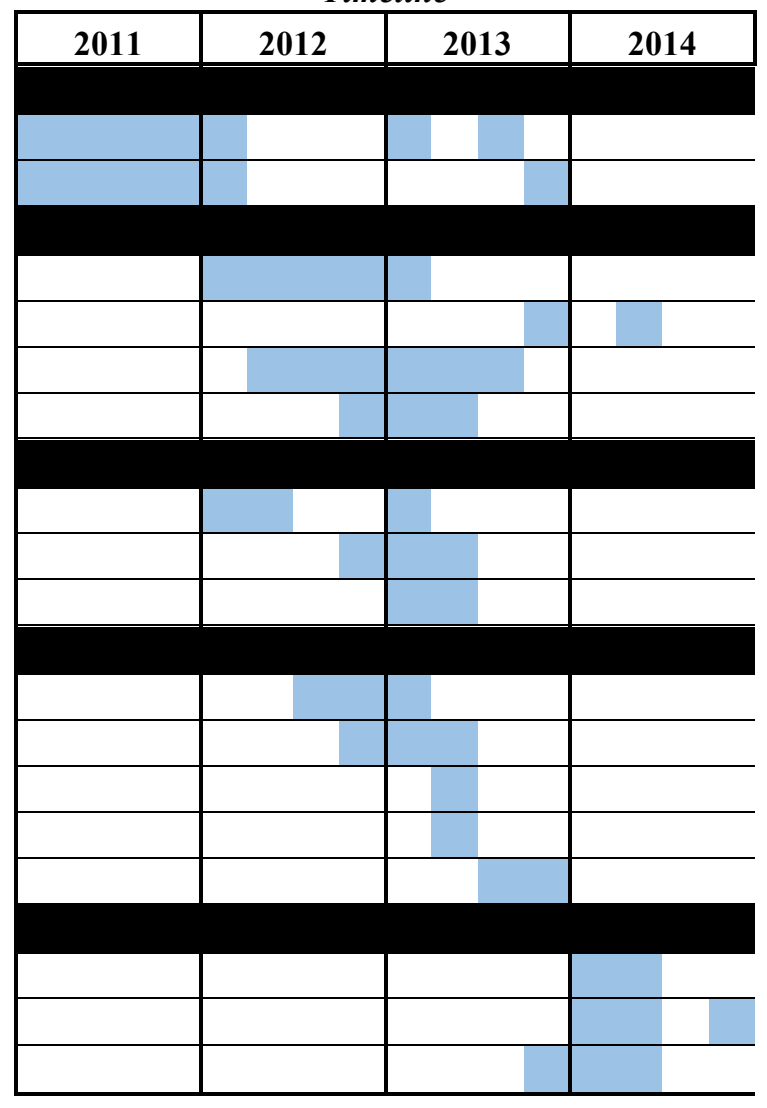


could be useful in rural clinics. This time target set the speed and power requirements of the centrifuge.

We could run the AMPS-based test in less than 15 minutes using a centrifuge capable of providing $13,700 \mathrm{~g}$; such a centrifuge requires power. For district and provincial hospitals in Zambia, power can be accessed from the grid. Many Zambians, however, receive their care from rural clinics. In rural clinics and villages, one can often find solar panels charging car batteries. To meet the needs of rural users, we used a DC-to-DC adapter to power the centrifuge with a car battery.

To ensure the test was easy to use, the entire process of the interaction of a patient with a diagnostic — from sample acquisition to reading the results — had to be carefully designed for ease of use and safety (Figure 5). We used polycarbonate capillary tubes, rather than glass tubes (avoided breakage, and the potential for puncture wounds). We designed the test so that tubes would be preloaded with solutions of polymers. Taking a sample of blood from a fingerstick and transferring it simply and practically, into a device is non-trivial. We iterated several designs (Figure 6) before finding a method — using capillary filling — to add a fixed volume of blood to a capillary that was already sealed on one end and partially filled with an AMPS solution. Visual readout removed the need for additional equipment or computing power. Including aspects of sample acquisition and readout into the design of the device ensured a smooth interaction with an end-user: all that a user would have to do would be to load a drop of blood, and spin the small test in the centrifuge.

\subsection{Validation confirmed that the technology was ready for field-trials}

Blood samples provided by our collaborators at Children's Hospital Boston allowed us to 
Figure 5. AMPS enable a simple, rapid test for SCD using density. (A) A capillary pre-loaded with AMPS wicks a fixed volume of blood into the device. After covering the hole for filling the tube with a sleeve, centrifugation enables the rapid sedimentation of cells over the AMPS and provides a result that can be read by eye. A red layer above the seal at the bottom of the tube indicates the presence of SCD. (B) The entire process of performing a test, including centrifugation, requires $\sim 12$ minutes. 


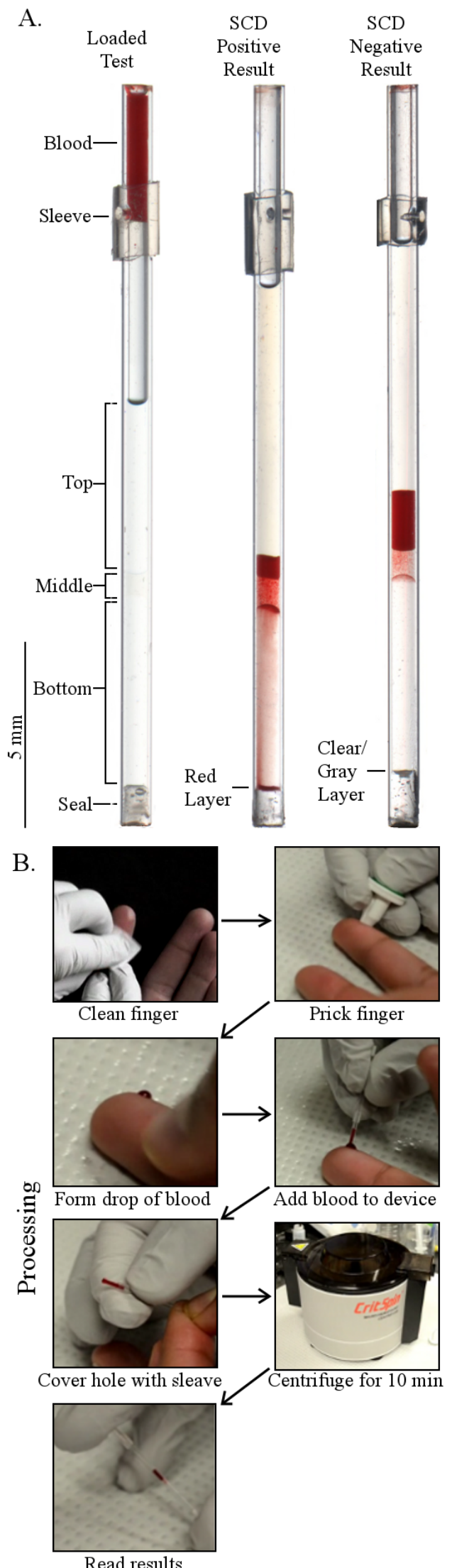

Read results 
Figure 6. Iterations on the design of the loading mechanism for a density-based SCD test. We tested three different methods to add blood to a capillary tube that was preloaded with AMPS and sealed on one end: 1) tube-in-tube method, 2) paper-in-tube method, and 3) hole-in-tube method. The tube-in-tube method allows a fixed volume of blood to be added to the larger tube loaded with AMPS, but requires dexterity to assemble. The paper-in-tube method uses the wicking ability of a thin strip of chromatography paper with large pore sizes to add whole blood into the tube by simply touching the open end to a drop of blood, but causes lysis upon centrifugation to remove the blood from the paper. The hole-in-tube method allows a fixed volume to enter the preloaded tube via capillary action, and a silicone sleeve covers the filling hole to prevent blood from leaking during centrifugation. 


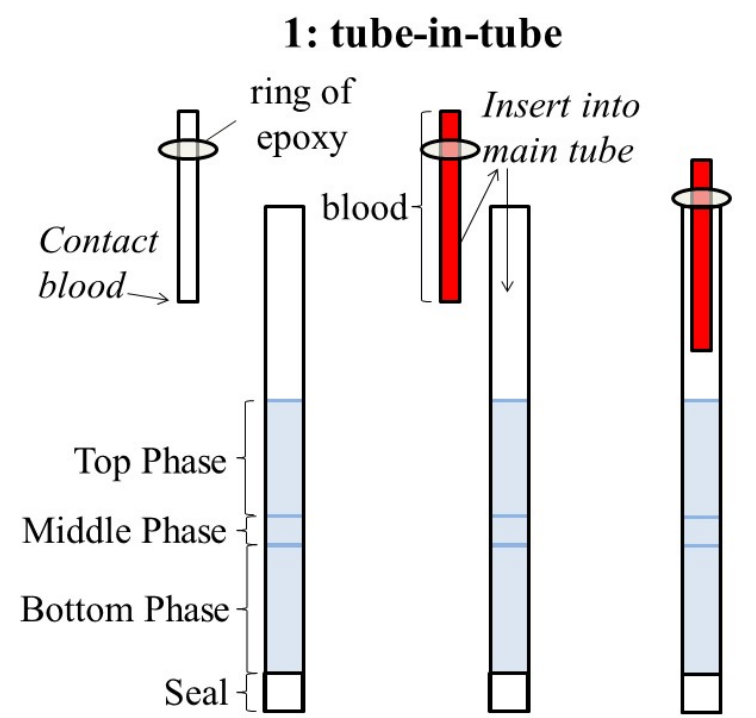

\section{2: paper-in-tube}
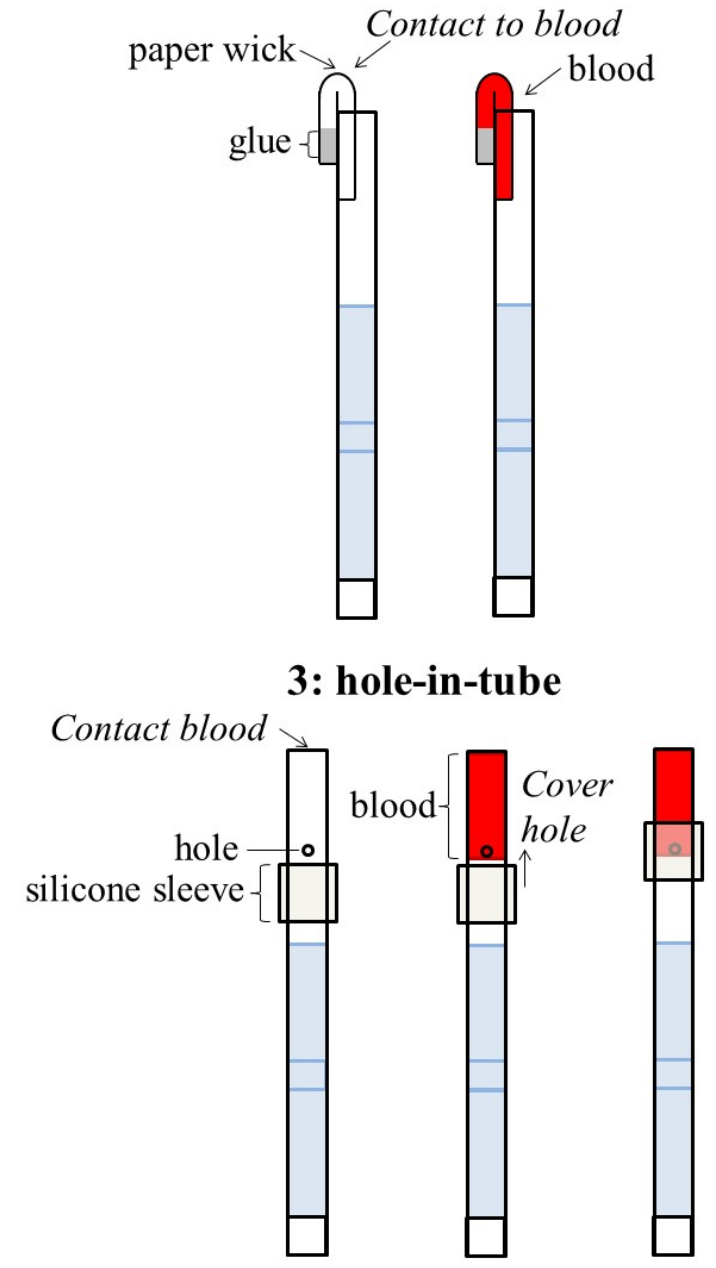
make rapid improvements on early iterations of the density-based test. Once densities of the AMPS were identified that discriminated between normal blood, and blood from individuals with sickle cell disease, the design was frozen and testing was carried out on a larger number of samples $(n=59)$. To achieve this sample size, we collaborated with Prof. Sergey Shevkoplyas, then at Tulane University, and Dr. Julie Kanter, then at the Sickle Cell Center of Southern Louisiana.

For the initial proof-of-principle, we included the main two genotypes of SCD (HbSS and $\mathrm{HbSC}$ ) and also tested sickle cell trait (HbAS) along with other non-sickle cell subjects (HbAA). Initial results showed a promising ability to discriminate between SCD and non-SCD with only 10 minutes of centrifugation and a drop $(\sim 5 \mu \mathrm{L})$ of blood. To enable the test to leave the bench, we also began work on the issues of packaging and storage. We used various packaging materials and accelerated storage tests in an oven to assess the risk for evaporation before settling on a packaging system for the evaluation in Zambia. ${ }^{[12]}$

\subsection{How funding was obtained}

Although the clinical need for a rapid test for SCD was clear from conversations with Dr. Stossel and Dr. Chunda-Liyoka, the way to fund such research was not apparent at first. In general, SCD suffers from a low level of funding, as is often the case with neglected tropical and orphan diseases. We began applying to a number of innovation awards focused on global health. One difficulty with these grants was pressure to have data from the field to justify the award. Research to provide such data, however, required funding. This "chicken-and-egg" scenario is characteristic of projects at the border between laboratory and field settings.

To obtain our initial funding for SCD, we had to think of other applications for the AMPS technology that would perhaps be more attractive to granting agencies. By proposing to 
use AMPS both to diagnose SCD and to also explore circulating tumor cells, we were able to put together a successful proposal to the Blavatnik Biomedical Accelerator Fund. This award supported the development of the test and the field trial at UTH. A smaller award from the Harvard Global Health Institute enabled us to perform an evaluation of the usability of the test with end-users in rural clinics in Zambia.

Our track record working with UTH and performing the field trial aided us in securing funding for a second field trial from the Consortium for Affordable Medical Technologies (CAMTech), a part of Massachusetts General Hospital's Center for Global Health.

\subsection{Designing the Study}

Dr. Stossel's connection to Zambia was one of the motivations for beginning work on a SCD diagnostic and, thus, work in Zambia was a natural choice for a field evaluation. Through Dr. Stossel, the team from Harvard connected with Dr. Chunda-Liyoka at UTH and began to draft grant applications, study protocols, and IRB submissions.

The site for the field evaluation was the Department of Paediatrics and the Department of Haematology at UTH in Lusaka, Zambia. The facilities at UTH could support equipment to do a gold-standard measurement of SCD (i.e., hemoglobin electrophoresis) and UTH also had a large population of SCD patients. UTH regularly monitors patients with SCD through a program that includes regular evaluation and follow-up. As a result of this program, over 3,000 patients with SCD are currently being managed by the hospital. Every Friday, roughly 50 of these patients receive follow-up.

The primary relevance of a rapid test for SCD is for use in children, and hence, the study only enrolled subjects under 18 years of age. Working with children required consent of a guardian as well as assent for children above a certain age. Literacy and language differences 
also added to the requirements of the consenting process to ensure adequate protection of subjects. Working in close consultation with both the local IRB in Zambia and the Committee on the Use of Human Subjects at Harvard was critical to ensure that all aspects of the ethics of the research were considered.

SCD screening at UTH relied primarily on solubility tests (e.g., Sickledex). Positive tests were followed up by gel electrophoresis. The electrophoresis equipment, however, was quite old and unreliable. Part of the study budget, therefore was allocated to update the clinical laboratory at UTH with a semi-automated hemoglobin electrophoresis unit (SAS1/2, Helena). In particular, this system allowed quantitative measurements of different hemoglobin types, including fetal hemoglobin $(\mathrm{HbF})$ - a parameter of interest for the density-based test. We also collected routine complete blood count (CBC) information on all subjects in the study and used a questionnaire to gather basic demographic information, and information about factors that might constitute a confounding factor for our method (i.e., recent sickle crisis).

Outside of the medical data, we also designed methods to record tests that had packaging failures, temperature on the days testing was performed, and the time between the blood draw and the use of rapid and gold-standard tests. Guidelines for the use of samples for various tests ( $\mathrm{CBC}$, hemoglobin electrophoresis, and the rapid test) were decided based on guidelines from the manufacturers or, in the case of the rapid test, experience from initial validation. Samples that were processed on any method outside the recommended timeline would be invalid. One variable that was overlooked in the design phase of this trial was the inclusion of temperature and humidity loggers during shipment.

Through the Harvard Catalyst program, the team consulted with a biostatistician to design the size of the cohorts to be recruited in the study. The trial was designed to recruit $\sim 600$ 
subjects over six months. The process of designing the study, obtaining IRB approval, and installing the new hemoglobin electrophoresis equipment took over nine months before the study could begin.

In addition to the evaluation of the performance of the test, we also wanted to evaluate the test in rural clinics for ease-of-use. Working with UTH and the U.S. Peace Corps in Zambia, we identified rural clinics to visit and designed a program to explain the test and receive feedback. The U.S.-based IRB committee declared the survey instrument to be exempt from full review as human subject research because of minimal risk and the nature of the information collected. The Zambian-based IRB committee, however, required a full review of the survey at the rural sites.

\subsection{Implementation}

A researcher from Harvard traveled to Lusaka at the beginning of the trial. Setting up the trial required a week. A full-day training of the study staff included the overall design of the study, workflow, recruitment, use of the rapid test, and management of data. Four readers were trained (two laboratory technicians and two nurses) using images of results of rapid tests and examples by an expert reader. A poster outlining each step of the use of the rapid test was placed prominently in the laboratory where rapid tests were run. A two-week pilot phase followed.

The pilot phase was critical for the success of the study. During this time, we evaluated initial concordance between the readers at UTH and the expert reader. We also identified and remedied potential problems with sample handling and workflow. For example, blood samples were collected in Vacutainers (Becton Dickinson) containing ethylenediaminetetraacetic acid (EDTA) as an anticoagulant. A fraction of each sample was transferred to a second tube. One 
tube of the sample went to the laboratory running hemoglobin electrophoresis while the other tube went to the laboratory running the rapid tests. Using separate laboratories aided with blinding the study. Initially, the second tube to which blood was added also contained EDTA. This additional EDTA could have caused dehydration of the cells and compromised the rapid test. This potential problem was identified during the pilot phase and all subsequent samples were aliquotted into untreated tubes after collection in anticoagulant treated tubes.

Halfway through the study, the expert reader from Harvard returned to the study site and performed a blinded test for concordance with three of the local Zambian readers. Throughout the study, the team at Harvard made batches of AMPS solutions and assembled packages (by hand) with hundreds of rapid tests to ship to the study site.

\subsection{Context and Culture}

Recruitment of subjects with SCD was generally much easier than recruitment of subjects without SCD because patients with the disease, and their parents, generally were more knowledgeable about the disease and the need for a rapid diagnostic test than the general population. Occasionally, parents chose not to participate in the study because there was concern about what could be done with the blood of their child. Some parents expressed a belief that a child has a finite amount of blood in their entire life and they were afraid that the child would not have blood left if they provided blood for the study in addition to a clinically indicated blood draw.

\subsection{Challenges}

Several unexpected obstacles threatened the completion of the study, but fortunately, all were overcome. At two points during the six month trial, nurses at UTH went on strike. Although the nurses on the study did not strike, their workload for their non-study obligations 
increased. The continued recruitment of subjects during this time is a testament to the commitment of these nurses to the study.

Near the middle of the study, a major fire broke out in the Nairobi International Airport in Kenya. Although far from the study site, this airport was part of the delivery route for the batches of AMPS tests shipped to Zambia. The fire occurred just after one batch had been shipped overseas and, as a result, that batch took an extra week to arrive at UTH. This extra shipping time, and the much elevated temperatures encountered by the tests as they awaited shipment through the damaged airport, could have compromised the tests, but unfortunately, the conditions during shipment were unknown. Indeed, the performance of the delayed batch was significantly worse than the other batches, ${ }^{[12]}$ but without additional information, we could not justify exclusion from the data analysis. This experience demonstrates the importance of setting clear parameters for valid results, including shipping and storage conditions, along with the use of temperature and humidity loggers to capture the relevant data.

Although the nurses' strike did not halt the study, supply-chain problems did cause a temporary pause. Initially, we purchased about $20 \%$ more electrophoresis gels than we believed would be required for the study. A fault in the heating unit in the electrophoresis instrument meant that only eight out of twelve lanes ran properly. Delays in getting a technician to fix the instrument meant that we ran out of gels before recruitment was complete. As a result, we suffered a one-month halt in the study while we waited for additional gels from the supplier. Even though we were not able to recruit the number of subjects that we had wanted due to the delays, we were still able to collect enough samples to retain statistical power before the budget for the study was spent. The dependence of the gold-standard device on maintenance and technical expertise highlighted the need for simplicity in design of diagnostics for the POC. 


\subsection{Lessons learned}

The details of the performance of the device and user feedback are described in detail elsewhere. ${ }^{[12]}$ Briefly, of the two prototypes tested, the best system had a diagnostic accuracy of $77 \%$. In general, false positives were more frequent than false negatives (more so than was found in the initial validation studies). Variation in performance between batches was fairly significant. We are actively working on improving quality controls and developing standards to use with the rapid tests to reduce the variability between batches. Concordance between readers was high, but could be improved by clearer guides for readers and more extensive training.

Apart from the technical knowledge gained from the field evaluation, we also gained significant contextual knowledge. The visit to the rural clinics was particularly informative. We were able to verify that appropriate interventions for SCD existed in rural clinics as well as off-grid access to power through solar panels charging car batteries.

Perhaps one of the most interesting outcomes of the field evaluation came to light during exit interviews of the study staff in Zambia by the Harvard team member. A number of staff members commented - without any specific prompting — that they had a new perspective when thinking about using diagnostics and developing technology for issues relevant to Zambia; if researchers abroad could come up with low-cost rapid tests, perhaps they- the Zambians themselves - could also come up with useful technologies. Although quantifying the impact of inspiration and modeling innovation is difficult, at best, the aspect of directly sharing knowledge and skills during such an international collaboration is a welcome side-effect of co-creation and field-evaluation.

\subsection{Next Steps}


Development of the next generation prototype of a rapid test for SCD is underway. Data from the field validation and initial work have enabled us to write new grants, and have sparked conversations with companies interested in developing the technology. Daktari Diagnostics, Inc. is assisting with the development of the test and was awarded a Small Business Innovation Research (SBIR) grant from the NIH to improve and test the device.

\section{Conclusions \& Recommendations}

\subsection{General lessons learned}

In both cases presented here, the technologies are not yet products, but field evaluation has significantly improved the POC devices, and has identified specific deficiencies still to be addressed. Our experiences suggest five summary lessons: 1) Begin planning and partnerships early. Ideally, partnerships should be in place before applying for grants. Long-standing partnerships allow clear communications that enable a team properly to identify a problem, and also to work through challenges of performing a field trial while maintaining good relations; 2) Get feedback early and often. Even if a full field trial is not appropriate for a particular stage of development of a device, getting feedback about the design and use of a device consistently throughout development will improve its quality and the chances of success in a large field trial; 3) Track every variable. No matter how much validation is done, there will be variables that are not accounted for in the technical specifications of a device. Even if every variable is not controlled, most variables can be measured and these data may prove to be the key to understanding the results from the field; 4) Aim for more subjects than the minimum needed. Unexpected problems can pause or end a study prematurely. Understanding the statistical power of a study and the amount the sample size could be reduced before the study loses value is critical. Within reason, aiming for more subjects than needed provides flexibility with the timing 
of the study. In our experience a buffer of $20-30 \%$ more subjects than required for the desired statistical power was appropriate; 5) Have patience. Taking the time to get IRB approval, defining every part of the protocol, working out the details of the supply chain, and doing a pilot study are all important steps to ensure that a field trial will be successful.

\subsection{From the Bench to the Field, and the Field to the Shelf}

Field evaluations are not only the purview of companies. Although a partnership between a not-for-profit company (DFA), with skill in bioengineering, and an academic group (the Whitesides lab), more capable in invention, developed the LFT, an NGO (PATH) led the field evaluation of the LFT device. An academic group (the Whitesides group), led the field evaluation of the AMPS-based rapid test for SCD with partners at UTH. In fact, the ability to demonstrate functionality (even imperfect functionality) in the field greatly reduces the risk in further development, and provides the group involved the chance to develop more of the resources needed for further work (through grants, collaborations, or transitions of the technologies to larger organizations).

Someday, we hope that both the LFT and the SCD-AMPS will be available on the shelves of clinics as finished products, but successful field trials are only steps along that path. When that day arrives that these technologies do become products, the experience and lessons of early field evaluations will have played an important role.

\section{Acknowledgements}

The initial, critical funding for this work was provided by the BMGF in a program designed together with Dr. Boris Nikolic. The authors thank Dr. Nira Pollock (BIDMC), Sarah McGray (PATH), Dr. Bernhard Weigl (PATH), Dr. Sarah Vella (Xerox), Dr. Andres Martinez 
(California Polytechnic), and Sariah Khormaee (Harvard Medical School) for helpful conversations and discussions. The initial work in paper-based diagnostics was carried out by Scott Phillips and Andres Martinez, and stimulated by work by Sam Sia and Vincent Linder.

A.A.K. acknowledges support from a Graduate Research Fellowship from the National Science Foundation. J.W.H. and B.S.S were supported by BMGF (51308).

\section{References}

[1] P. Yager, G. J. Domingo, J. Gerdes, Annu. Rev. Biomed. Eng. 2008, 10, 107-144.

[2] S. K. Sia, L. J. Kricka, Lab Chip 2008, 8, 1982-1983.

[3] C. D. Chin, V. Linder, S. K. Sia, Lab Chip 2007, 7, 41-57.

[4] C. D. Chin, V. Linder, S. K. Sia, Lab Chip 2012, 12, 2118-2134.

[5] G. Whitesides, Lab Chip 2014, 14, 3125-3126.

[6] D. A. Pitta, R. Guesalaga, P. Marshall, J. Consum. Mark. 2008, 25, 393-401.

[7] A. W. Martinez, S. T. Phillips, M. J. Butte, G. M. Whitesides, Angew. Chem. Int. Ed. Engl. 2007, 46, 1318-1320.

[8] C. R. Mace, O. Akbulut, A. A. Kumar, N. D. Shapiro, R. Derda, M. R. Patton, G. M. Whitesides, J. Am. Chem. Soc. 2012, 134, 9094-9097.

[9] N. R. Pollock, J. P. Rolland, S. Kumar, P. D. Beattie, S. Jain, F. Noubary, V. L. Wong, R. A. Pohlmann, U. S. Ryan, G. M. Whitesides, et al., Sci. Transl. Med. 2012, 4, 152 ra129.

[10] A. A. Kumar, M. R. Patton, J. W. J. W. Hennek, S. Y. R. S. Y. R. Lee, G. D’Alesio-Spina, X. Yang, J. Kanter, S. S. Shevkoplyas, C. Brugnara, G. M. Whitesides, et al., Proc. Natl. Acad. Sci. U. S. A. 2014, 111, 14864-14869.

[11] N. R. Pollock, S. McGray, D. J. Colby, F. Noubary, H. Nguyen, T. A. Nguyen, S. Khormaee, S. Jain, K. Hawkins, S. Kumar, et al., PLoS One 2013, 8, e75616.

[12] A. A. Kumar, C. Chunda-Liyoka, J. W. Hennek, H. Mantina, S. Y. R. Lee, M. R. Patton, P. Sambo, S. Sinyangwe, C. Kankasa, C. Chintu, et al., PLoS One 2014, doi:10.1371/journal.pone.0114540. 
[13] This criterion may be less important in cases where incentives are created, either by government initiatives or non-profit organizations working to promote research on niche diseases. For example, the Food and Drug Administration (FDA) in the US has promoted research on orphan diseases through various incentives (http://www.fda.gov/forindustry/DevelopingProductsforrareDiseasesConditions/default.ht $\mathrm{m})$.

[14] R. W. Peeling, D. Mabey, Clin. Microbiol. Infect. 2010, 16, 1062-1069.

[15] D. Mabey, R. W. Peeling, A. Ustianowski, M. D. Perkins, Nat. Rev. Microbiol. 2004, 2, 231-240.

[16] H. Kettler, K. White, S. Hawkes, Mapping the Landscape of Diagnostics for Sexually Transmitted Infections: Key Findings and Recommendations, 2004, Available at: http://www.who.int/tdr/publications/documents/mapping-landscape-sti.pdf.

[17] eMarketer Inc., "Smartphone Users Worldwide Will Total 1.75 Billion in 2014," can be found under http://www.emarketer.com/Article/Smartphone-UsersWorldwide-Will-Total175-Billion-2014/1010536, 2014.

[18] N. P. Pai, C. Vadnais, C. Denkinger, N. Engel, M. Pai, PLoS Med. 2012, 9, e1001306.

[19] B. H. Weigl, D. S. Boyle, T. de los Santos, R. B. Peck, M. S. Steele, Expert Rev. Med. Devices 2009, 6, 461-464.

[20] B. A. Caldwell, A. Young, J. Gomez-Marquez, K. R. Olson, IEEE Pulse 2011, 63-67.

[21] C. VanVoorhis, B. Morgan, Tutor. Quant. Methods Psychol. 2007, 3, 43-50.

[22] G. Cumming, F. Fidler, D. L. Vaux, J. Cell Biol. 2007, 177, 7-11.

[23] D. Vaux, Nature 2013, 492, 8-9.

[24] National Committee for Clinical Laboratory Standards, Procedures and Devices for the Collection of Diagnostic Capillary Blood Specimens (Approved Standard HA-A5), NCCLS, Wayne, PA, 2004.

[25] C. Metz, Semin. Nucl. Med. 1978, VIII, 283-298.

[26] J. M. Bland, D. G. Altman, Stat. Methods Med. Res. 1999, 8, 135-160.

[27] S. J. Pocock, L. M. Friedman, C. D. Furberg, D. L. Demets, Fundamentals of Clinical Trials, Springer, New York, 2010.

[28] R. Glasgow, D. Magid, A. Beck, Med. Care 2005, 43, 551-557. 
[29] J. Lachin, Control. Clin. Trials 1981, 113, 93-113.

[30] K. S. Button, J. P. A. Ioannidis, C. Mokrysz, B. A. Nosek, J. Flint, E. S. J. Robinson, M. R. Munafò, Nat. Rev. Neurosci. 2013, 14, 365-376.

[31] United Nations, Political Declaration on HIV and AIDS: Intensifying Our Efforts to Eliminate HIV and AIDS, 2011, Available at: http://www.unaids.org/sites/default/files/sub_landing/files/20110610_UN_A-RES-65277_en.pdf.

[32] E. Martínez, J. L. Blanco, J. A. Arnaiz, J. B. Pérez-Cuevas, A. Mocroft, A. Cruceta, M. A. Marcos, A. Milinkovic, M. A. García-Viejo, J. Mallolas, et al., AIDS 2001, 15, 12611268.

[33] Panel on Antiretroviral Guidelines for Adults and Adolescents., Guidelines for the Use of Antiretroviral Agents in HIV-1-Infected Adults and Adolescents., 2009, Available at: http://aidsinfo.nih.gov/ContentFiles/AdultandAdolescentGL.pdf.

[34] G. Meintjes, G. Maartens, A. Boulle, F. Conradie, E. Goemaere, E. Hefer, D. Johnson, M. Mathe, Y. Moosa, R. Osih, et al., South. Afr. J. HIV Med. 2012, 13, 36-45.

[35] S. J. McPhee, M. A. Papadakis, Eds., Current Medical Diagnostics and Treatment, McGraw Hill, New York, 2010.

[36] S. J. Vella, P. Beattie, R. Cademartiri, A. Laromaine, A. W. Martinez, S. T. Phillips, K. A. Mirica, G. M. Whitesides, Anal. Chem. 2012, 84, 2883-2891.

[37] A. W. Martinez, S. T. Phillips, G. M. Whitesides, E. Carrilho, Anal. Chem. 2010, 82, 310.

[38] A. W. Martinez, S. T. Phillips, E. Carrilho, S. W. Thomas, H. Sindi, G. M. Whitesides, Anal. Chem. 2008, 80, 3699-3707.

[39] A. W. Martinez, S. T. Phillips, G. M. Whitesides, Proc. Natl. Acad. Sci. U. S. A. 2008, 105, 19606-19611.

[40] C. D. Chin, T. Laksanasopin, Y. K. Cheung, D. Steinmiller, V. Linder, H. Parsa, J. Wang, H. Moore, R. Rouse, G. Umviligihozo, et al., Nat. Med. 2011, 17, 1015-1019.

[41] F. B. Piel, S. I. Hay, S. Gupta, D. J. Weatherall, T. N. Williams, PLoS Med. 2013, 10, e1001484.

[42] J. Hankins, R. E. Ware, Lancet 2009, 374, 1308-1310.

[43] M. Gaston, J. Verter, G. Woods, C. Pegelow, J. Kelleher, G. Presbury, H. Zarkowsky, E. Vichinsky, R. Iyer, J. Lobel, et al., N. Engl. J. Med. 1986, 314, 1593-1599. 
[44] M. E. Fabry, J. G. Mears, P. Patel, K. Schaefer-Rego, L. D. Carmichael, G. Martinez, R. L. Nagel, Blood 1984, 64, 1042-1046.

[45] S. Embury, M. Clark, J. Clin. Invest. 1984, 73, 116-123.

[46] R. C. Leif, J. Vinograd, Proc. Natl. Acad. Sci. U. S. A. 1964, 51, 520-528.

[47] W. H. Grover, A. K. Bryan, M. Diez-Silva, S. Suresh, J. M. Higgins, S. R. Manalis, Proc. Natl. Acad. Sci. U. S. A. 2011, 108, 10992-10996.

[48] H. B. Weems, L. S. Lessin, Acta Haematol. 1984, 71, 361-370.

[49] A. A. Kumar, C. Lim, Y. Moreno, C. R. Mace, A. Syed, D. Van Tyne, D. F. Wirth, M. T. Duraisingh, G. M. Whitesides, Am. J. Hematol. 2014, DOI: 10.1002/ajh.23860.

[50] C. Brugnara, T. Van Ha, D. C. Tosteson, Blood 1989, 487-495.

[51] C. Brugnara, J. Pediatr. Hematol. Oncol. 2003, 25, 927-933.

[52] A. Kratz, M. Ferraro, P. M. Sluss, K. B. Lewandrowski, N. Engl. J. Med. 2004, 351, $1548-1564$. 


\section{PERSPECTIVE}

This paper shares lessons from two case studies in the development of point-of-care tests: (1) a colorimetric, paper-based liver function test using serum transaminases, and (2) a test for sickle cell disease using aqueous multiphase systems and differences in the density of red blood cells characteristic of sickle cell disease.

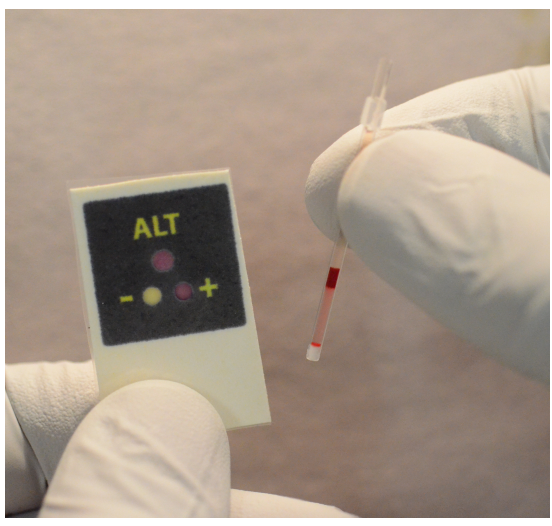

Ashok A. Kumar, Jonathan W. Hennek, Barbara S. Smith, Shailendra Kumar, Patrick Beattie, Sidhartha Jain, Jason P. Rolland, Thomas P. Stossel, Catherine Chunda-Liyoka, and George M. Whitesides*

Page No. - Page No.

From the Bench to the Field in LowCost Diagnostics

Keywords: point-of-care diagnostics; technology translation; field trials; liver injury; sickle cell disease 